\title{
MEJORAS DEL SIMULADOR DE DISPERSIÓN DE GASES GADEN PARA DESARROLLOS DE ROBÓTICA OLFATIVA
}

\author{
Pepe Ojeda, Javier Monroy y Javier Gonzalez-Jimenez \\ Dpto. Ingeniería de Sistemas y Automática, Instituto de Investigación Biomédica de Málaga (IBIMA), \\ Universidad de Málaga, 29071, Málaga, España. \\ \{ojedamorala||jgmonroy||javiergonzalez\}@uma.es
}

\begin{abstract}
Resumen
Dentro de las disciplinas cientificas, las herramientas de simulación juegan un papel fundamental durante el proceso de diseño y desarrollo de nuevas soluciones, permitiendo reducir los tiempos de desarrollo y posibilitando un estudio detallado del efecto de múltiples parámetros. En este trabajo nos centramos en GADEN, un novedoso simulador de dispersión de gases para aplicaciones robóticas, proponiendo un conjunto de mejoras con el objetivo de facilitar su uso y mejorar su rendimiento computacional. Además de una descripción detallada de los cambios realizados, se incluye un estudio cuantitativo del efecto de los mismos sobre el rendimiento de la simulación.
\end{abstract}

Palabras clave: GADEN, ROS, dispersión de gases, robótica olfativa, entorno de simulación, búsqueda de fuentes, mapas de distribución.

\section{Introducción}

El término "robótica olfativa" hace referencia al campo de investigación centrado en combinar sistemas robóticos inteligentes con el sentido del olfato artificial mediante la integración de las denominadas narices electrónicas [5, 9, 25]. Los importantes desarrollos tecnológicos de la última década en el diseño y fabricación de las narices electrónicas, así como los continuos avances en la aplicación y adaptación de técnicas de procesado de señal y aprendizaje automático a este campo $[3,16,17]$, permiten en la actualidad poder afrontar una gran variedad de aplicaciones prácticas. Entre estas aplicaciones cabe destacar la búsqueda y localización de fuentes emisoras de gases (e.g. fugas, escapes, puntos de emisión, etc.) $[7,10,20,26]$, la creación de mapas de concentración que permitan analizar como los gases se distribuyen en el entorno $[11,14,19,22]$, o la propia clasificación del tipo de sustancia gaseosa a la que el robot está siendo expuesto $[15,23,29]$.

La robótica olfativa es un campo emergente y de rápida evolución [8] con importantes desafíos científico-técnicos aun por abordar, entre ellos, la

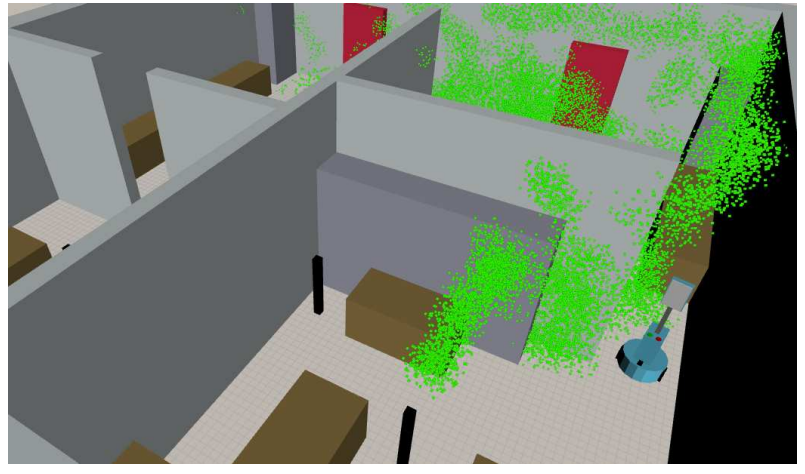

Figura 1: Ejemplo de una simulación de dispersión de gases en GADEN. Como se aprecia, GADEN integra la dispersión de gases (verde) en entornos realistas en $3 \mathrm{D}$, junto con sistemas robóticos.

dificultad de comparar y evaluar experimentalmente diferentes métodos. Este problema se debe a la imposibilidad de obtener un ground truth de la dispersión de los gases liberados durante una sesión experimental [28], y por consiguiente la complejidad a la hora de evaluar la eficiencia de un nuevo algoritmo.

Dos propuestas han sido tradicionalmente empleadas para paliar este importante problema: (i) la simplificación de las condiciones experimentales para poder obtener dispersiones de gas controladas (e.g. controlar las condiciones de viento con ventiladores, el número y localización de las emisiones de gas, limitar el tamaño del área de trabajo a pocos metros cuadrados, o eliminar cualquier objeto que pudiera influir en la dispersión de los gases) y (ii) el uso de entornos de simulación (GDS, del inglés Gas Dispersion Simulator).

La primera opción, aunque ampliamente adoptada durante las últimas décadas, conlleva la imposibilidad de poder evaluar la eficiencia de los métodos propuestos en condiciones reales, dónde, por ejemplo, no se pueden controlar las condiciones medioambientales, o dónde el robot trabaja en presencia de objetos y obstáculos (e.g. paredes, mobiliario, otros robots, etc.). La segunda opción, la cual está ganando popularidad en los últimos años, permite eliminar está limitación al considerar entornos más complejos y por tanto más cercanos a los casos de uso previstos. No obstante, 
el inmaduro estado actual de las herramientas de simulación para la robótica olfativa hace necesario que en la mayoría de casos se tengan que incluir tanto experimentos simulados en entornos complejos, como experimentos reales "simplificados". Por tanto, la mejora de los entornos de simulación disponibles para la robótica olfativa, que faciliten estas tareas de evaluación y experimentación, resulta de gran interés.

Entre las herramientas disponibles destaca GADEN [18], un simulador de dispersión de gases diseñado específicamente para el desarrollo de aplicaciones robóticas (Figura 1). En este trabajo se presentan nuevas funcionalidades que lo hacen más accesible, con intención de difundir su uso entre la comunidad científica, así como diversas mejoras que se han llevado a cabo para optimizar su rendimiento.

\section{Trabajos Relacionados}

Esta sección analiza los principales simuladores de dispersión de gases, haciendo especial hincapié en aquellos que posibilitan, en mayor o menor medida, una integración con sistemas de simulación robóticos, esto es, que permitan evaluar y testear algoritmos de robótica olfativa (e.g. búsqueda de fuentes, generación de mapas, etc.).

En la actualidad existen diferentes herramientas para la simulación de dispersión de gases. Por una parte encontramos proyectos como ANSYS $^{1}$ u OpenFOAM ${ }^{2}$, que proponen simuladores desde un punto de vista físico-teórico a través de la resolución computacional de las ecuaciones que controlan la dinámica de fluidos (CFD, del inglés Computational Fluid Dynamics). Estas herramientas permiten el uso de modelos de dispersión sofisticados y precisos que se ajustan bien a la realidad incluso en entornos complejos [24,27]. No obstante, este tipo de simulador no está diseñado para aplicaciones de robótica olfativa, por lo que no permiten la simulación de sensores para la medición de la concentración del gas o del vector de viento, ni ofrecen una interfaz de integración adaptada a las plataformas robóticas. Estos problemas, junto con el expertise necesario para ajustar los múltiples parámetros de este tipo de herramienta, limitan en gran medida su aplicación y difusión en este campo.

Por otro lado, existen propuestas de simuladores de dispersión de gases más enfocadas a la robótica olfativa. Entre estas, cabe destacar herramientas como PlumeSim [2], desarrollado para el proyecto Player/Stage [21], o el simulador de dispersión

\footnotetext{
${ }^{1}$ https: //www.ansys.com/

${ }^{2}$ https : / /WWw.openfoam. com/
}

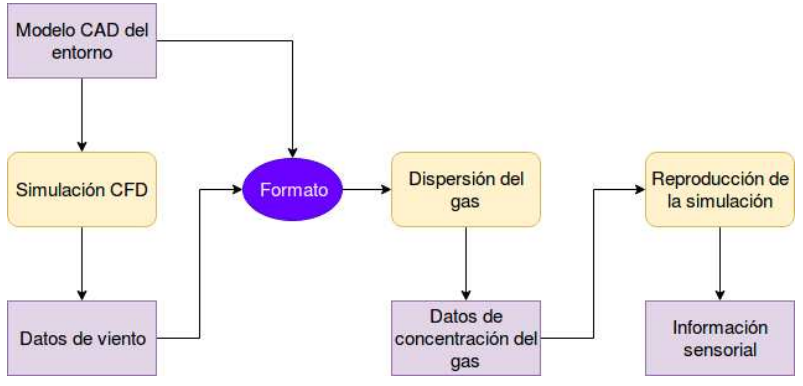

Figura 2: Estructura de GADEN y flujo de datos en el proceso de simulación.

de gases basado en OpenMora [13]. Ambas propuestas integran el uso de modelos de dispersión mucho más simplificados (como el caso de plumas gaussianas [12]), permitiendo una fácil evaluación de diferentes estrategias en entornos simples, o la posibilidad de incorporar datos de la simulación de los gases obtenidos externamente a través de otras herramientas de simulación (por ejemplo basados en CFD). A pesar de su mayor flexibilidad, estas herramientas presentan importantes limitaciones como el hecho de ser simuladores que sólo trabajan en dos dimensiones (lo cual limita su extensión a casos reales) o la falta de integración con herramientas robóticas actuales, quedando estas obsoletas.

Atendiendo a estas limitaciones y a la necesidad de disponer de un entorno de simulación que permita integrar de forma eficiente la dispersión de gases con sistemas robóticos, surgió el proyecto GADEN $^{3}$ [18]. Este proyecto propone un novedoso simulador de dispersión de gases adaptado a las necesidades de la robótica olfativa desarrollado en el ampliamente utilizado sistema operativo $\mathrm{ROS}^{4}$. Este simulador se basa en el uso de herramientas de CFD para la simulación de los flujos de viento en $3 \mathrm{D}$, y la implementación de la dispersión del gas a través de la teoría de filamentos [4]. Dado que el presente trabajo se basa en esta herramienta, en la siguiente sección se presenta una descripción detallada de las funcionalidades, estructura y limitaciones de este interesante simulador.

\subsection{GADEN}

GADEN [18] ofrece una solución eficaz a las más importantes limitaciones de los simuladores anteriormente mencionados. Las simulaciones son tridimensionales, y permite definir entornos complejos de manera sencilla utilizando modelos CAD. Se basa en el uso de simulaciones CFD, que permiten un alto grado de precisión pero tienen el inconveniente de un alto coste computacional. Para

\footnotetext{
${ }^{3}$ http://mapir.isa.uma.es/work/gaden

${ }^{4}$ https: //www.ros.org
} 


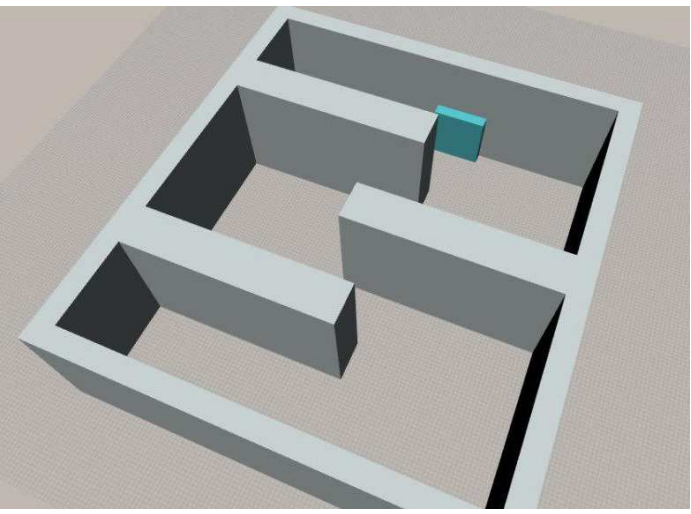

(a) El entorno es definido mediante uno o más modelos CAD.

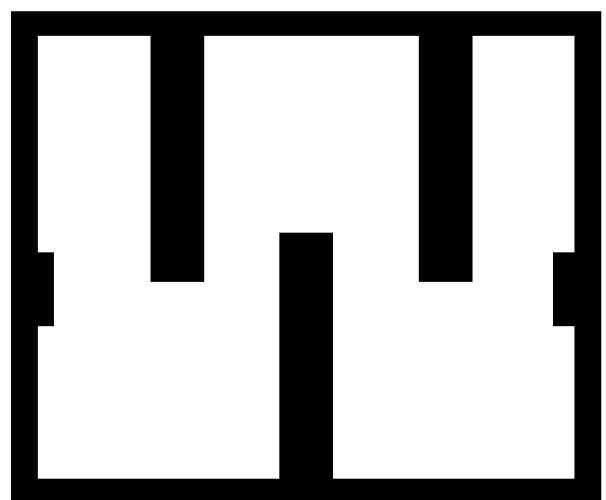

(b) Sección horizontal del mapa de ocupación generado a partir del modelo CAD. Este mapa es la entrada que espera el simulador.

Figura 3: Definición del entorno para la simulación.

solucionar este problema, GADEN lleva a cabo la simulación de viento y gas por adelantado, y guarda las condiciones resultantes en cada instante de tiempo. De tal manera, a la hora de utilizar el simulador para la experimentación con un robot, es posible reproducir la simulación sólo leyendo los datos guardados, lo cual conlleva un coste computacional mucho menor. Las simulaciones en GADEN se componen de tres procesos secuenciales (Figura 2).

(i) En primer lugar se realiza una simulación de CFD de manera externa para obtener los datos del viento en el entorno. Para definir el entorno se utiliza un modelo CAD. Tanto los datos del viento como el modelo CAD deben someterse a una fase de pre-procesamiento para darles el formato adecuado que permita utilizarlos en el siguiente paso (Figura 3).

(ii) Los datos de viento producidos en el paso anterior y el modelo CAD del entorno son utilizados para simular la dispersión de un gas, permitiendo controlar la posición y las características de la fuente que lo emite. Se utiliza el modelo de filamentos [4], creando nuevos filamentos según el ritmo de emisión establecido para la fuente, actualizando la posición y difusión de los filamentos existentes de acuerdo a las condiciones de viento y del entorno. Además, se eliminan los filamentos que alcanzan las zonas marcadas como salidas y se guarda la concentración del gas presente en cada una de las celdas, calculada en función de la posición de los filamentos.

(iii) Por último, las simulaciones se reproducen a partir de los datos generados por el paso anterior. Como se ha mencionado anteriormente, la principal ventaja de reproducir las simulaciones es el considerablemente menor coste computacional, que nos permite utilizar esta fase para el desarrollo de aplicaciones robóticas evitando la lentitud del proceso de simulación.

GADEN está implementado como un metapaquete de ROS, y los procesos que componen la simulación están separados en distintos paquetes. Entre las funcionalidades implementadas en ellos, además de las centrales que ya han sido comentadas, se encuentran un paquete ROS para facilitar la visualización del entorno y varios sensores simulados (MOX, PID, anemómetro 2D).

A pesar de ser uno de los simuladores más recientes y avanzados para el desarrollo y evaluación de algoritmos de robótica olfativa, GADEN aún presenta diferentes limitaciones o aspectos mejorables que han sido propuestos como lineas de trabajo futuro en pro de facilitar su difusión entre la comunidad científica. Entre ellos cabe destacar:

- Facilidad de uso. Los datos de entrada sobre los que trabaja GADEN (e.g. estructura del entorno, datos simulados del viento y carácteristicas de la/s fuente/s) requieren un formato específico y no estándar. Tal y como se aprecia en la figura 2, esta limitación hace necesario el uso de una fase de preprocesamiento de datos. En la versión actual, GADEN requiere de un script de Matlab para dicha tarea, requiriendo por tanto de herramientas externas y añadiendo complejidad extra a la tarea de definir nuevas simulaciones.

- Coste computacional. La simulación de la dispersión del gas, aunque sólo necesite realizarse una vez, es un proceso muy lento, ya que en cada paso es necesario calcular el movimiento de cada uno de los filamentos de gas 
y, posteriormente, el efecto que la posición de los filamentos tiene sobre la concentración de gas presente en cada una de las celdas.

- Memoria utilizada. Puesto que, para poder recrear las simulaciones, se registran las condiciones de viento y gas en cada una de las celdas del entorno cada pocas décimas de segundo, los resultados de las simulaciones ocupan una gran cantidad de memoria. Además, cabe mencionar que el número de celdas a considerar crece cúbicamente con las dimensiones del entorno de trabajo, aspecto que limita el uso de esta herramienta en entornos considerablemente grandes.

- Integración del robot en la simulación. El efecto de los movimientos del robot en el flujo del aire y la dispersión de los gases no es tenido en cuenta. Cabe señalar que esta limitación está presente en todos los simuladores mencionados.

\section{Mejoras Implantadas}

El objetivo final del presente trabajo es contribuir en la mejora de las características del simulador GADEN, haciéndolo más versátil y fácil de usar. Esto último contribuirá, igualmente, a una mejor difusión de dicha herramienta entre la comunidad científica con interés en la robótica olfativa. Para ello, en esta sección se describe una serie mejoras que solventan o palían algunas de las limitaciones actuales de dicha herramienta de simulación. Más concretamente, se proponen las siguientes contribuciones:

- Creación de una fase de pre-procesamiento automático de los datos de entrada (implementado como un nodo ROS), eliminando la necesidad de su pre-procesamiento manual a través de herramientas externas y mejorando la integración con modelos CAD.

- Mejora del rendimiento mediante la paralelización del código encargado de la dispersión del gas (teoría de filamentos).

- Reducción del tamaño de los conjuntos de datos generados, eliminando información redundante, optimizando el formato utilizado y aplicando técnicas de compresión.

\subsection{Pre-procesamiento de Datos}

Tal y como se describe en la figura 2, GADEN requiere como entrada dos conjuntos de datos: un mapa tridimensional del entorno en el que se indique qué zonas están libres y qué zonas están ocupadas (i.e. mapa de ocupación), y la información del viento en dicho entorno (entendido como el valor del vector de viento en cada una de las celdas que componen el entorno 3D). Este conjunto de datos necesita ser formateado para poder ser integrado en GADEN, lo cual hace necesaria una fase de pre-procesamiento. En esta sección se describe la propuesta implementada para realizar esta tarea de forma automática y conveniente para el usuario, facilitando considerablemente la definición de nuevos entornos de simulación.

\subsubsection{Integración de Datos de Viento}

El proyecto GADEN propone la simulación de las condiciones de viento mediante la herramienta de simulación de fluidos OpenFOAM, un proyecto de código abierto que permite definir el entorno al que queremos aplicar la simulación de CFD mediante una malla de polígonos (polymesh), que puede ser fácilmente generada a partir de un modelo CAD. Los datos del viento generados por OpenFOAM pueden ser exportados en forma de una nube de puntos (correspondientes a los centros de las celdas irregulares en las que se ha dividido el entorno), siendo cada punto interpretado como el correspondiente vector tridimensional de viento.

Este formato de datos, típico de las herramientas CFD, presenta un importante problema a la hora de ser integrado con GADEN. Con la intención de optimizar los cálculos y hacer más legibles los resultados de las simulaciones, GADEN emplea celdas tridimensionales perfectamente cúbicas y de tamaño constante. Este aspecto imposibilita una integración directa, y hace necesaria una fase de pre-procesado para encajar las celdas irregulares en la estructura regular.

Concretamente, el proceso se basa en calcular los índices de la celda cúbica a la que pertenece el centro de cada una de las celdas irregulares generadas por OpenFOAM. Teniendo en cuenta la diversidad de entornos a simular, la posible diferencia en la resolución y número de celdas de cada cada conjunto de celdas, y la presencia de obstáculos (en las que las condiciones de viento no se pueden calcular), se aprecia fácilmente que esta tarea no está exenta de complicaciones.

Para determinar a qué celda pertenece cada uno de los puntos proporcionados definimos la función indices:

$$
\text { indices }\left(\begin{array}{l}
p_{x} \\
p_{y} \\
p_{z}
\end{array}\right)=\left(\begin{array}{l}
\left(p_{x}-\min _{x}\right) / t_{c e l d a} \\
\left(p_{y}-\min _{y}\right) / t_{c e l d a} \\
\left(p_{z}-\min _{z}\right) / t_{c e l d a}
\end{array}\right)
$$

donde $p$ es el punto que aparece en los resultados de OpenFOAM, $\left(\min _{x}, \min _{y}, \min _{z}\right)$ son las coordenadas en las que comienza el entorno, y $t_{c e l d a}$ 


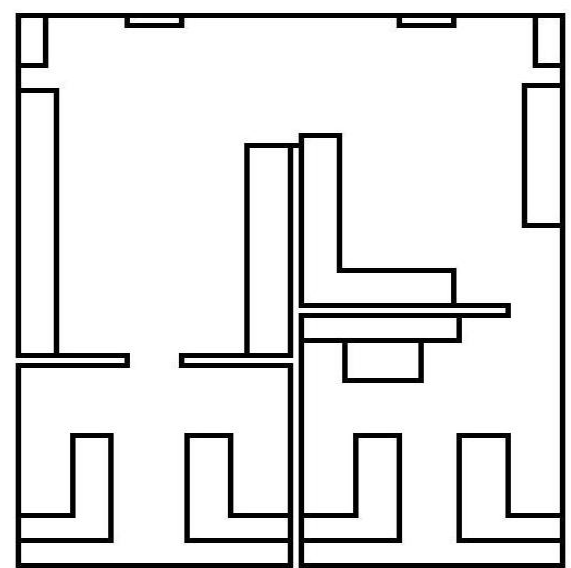

(a) Sección horizontal del mapa sólo con las superficies de los objetos.

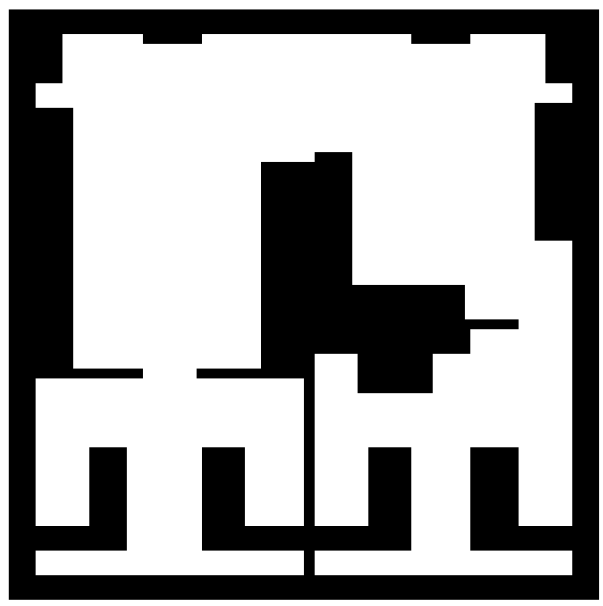

(b) Sección horizontal del mapa final.

Figura 4: Secciones de los mapas de ocupación generados.

es el tamaño del lado de las celdas.

Utilizando esta función, cada uno de los vectores de viento proporcionados por OpenFOAM es asignado a la celda que le corresponde.

\subsubsection{Mapa de Ocupación}

El otro requerimiento previo de GADEN, el mapa de ocupación, requiere un procesamiento más complejo. En la versión actual de GADEN, la situación de las celdas se extrapola a partir de la información del viento. Es decir, tras llevar a cabo el paso anterior y asignar la nube de puntos a sus correspondientes celdas, se considera que una celda está libre si alguno de los vectores de viento ha sido asignado a ella, y que está ocupada en caso contrario.

Esta aproximación resulta muy sencilla de implementar, y puede dar buenos resultados en caso de que las dimensiones de los objetos del mapa sean múltiplos del tamaño de las celdas, pero incluso en ese caso es falible. Para obtener un mapa fiable que el usuario no tenga que modificar manualmente, se ha implementado un algoritmo que lo genera de manera automática a partir de uno o más modelos CAD. Puesto que estos modelos ya han sido necesarios previamente para realizar la simulación del flujo de viento, no se requiere más trabajo por parte del usuario.

Aunque ha sido diseñado para GADEN, el algoritmo a continuación descrito puede utilizarse para generar mapas de ocupación tridimensionales para cualquier otra aplicación que los requiera. Concretamente, el algoritmo utiliza como entrada modelos CAD en formato STL, permitiendo usar cualquier número de ellos para definir el entorno, y requiriendo uno separado que indique dónde están las salidas por las que el gas puede escapar del sistema. En este formato, las superficies de los objetos se descomponen en triángulos, dando para cada uno de ellos la lista de las coordenadas de los vértices $\left(v_{1}, v_{2}, v_{3}\right)$, y el vector normal al plano $\Pi$ en el que se encuentra.

Una vez se ha leído el archivo y almacenado en una estructura de datos apropiada los datos de los triángulos, cada uno de ellos es tratado de manera independiente (puesto que el formato no indica si varios triángulos forman parte del mismo objeto). Para cada triángulo, se considera el conjunto $C$ de todas las celdas que forman parte del mínimo prisma rectangular que contiene al triángulo (2).

$$
\begin{aligned}
C= & \left\{\operatorname{celd}_{i, j, h} \mid x_{\min } \leq i \leq x_{\max }\right. \\
& \left.y_{\min } \leq j \leq y_{\max }, z_{\min } \leq h \leq z_{\max }\right\}
\end{aligned}
$$

donde $x_{\text {min }}$ es el mínimo valor de la primera componente de índices $\left(v_{1}\right)$, indices $\left(v_{2}\right)$ e índices $\left(v_{3}\right)$ (1); y $x_{\max }$ es el máximo. Las variables $y_{\min }$, $y_{\max }, z_{\min }, z_{\max }$ son análogas para las dos componentes restantes.

Cada una de las celdas pertenecientes a $C(2)$ se somete a dos comprobaciones para determinar si debe ser marcada como ocupada:

- La proyección del centro de la celda, o de alguno de sus vértices, sobre el plano $\Pi$ debe estar dentro del triángulo. Esta comprobación puede hacerse utilizando las coordenadas baricéntricas [6] de la proyección.

- El plano $\Pi$ debe pasar a través de la celda. Para comprobarlo, se calcula la distancia con signo de cada uno de los vértices de la celda 
al plano. Si hay al menos un vértice en cada lado del plano, el plano pasa a través de la celda. Esta condición también se considera satisfecha si alguno de los vértices pertenece al plano.

Dado que el formato con el que trabajamos sólo nos proporciona información sobre las superficies de los objetos, tras llevar a cabo este procedimiento para cada uno de los triángulos definidos en el archivo el mapa generado mostrará como ocupadas sólo las celdas en las que se encuentren las superficies de los objetos, y no las que correspondan al interior de los mismos (Figura 4a).

Aunque esto no debe causar problemas en las simulaciones, ya que GADEN comprueba todas las celdas en la trayectoria de un filamento de gas, se ha añadido un paso adicional al algoritmo para diferenciar el espacio vacío del interior de los objetos. Consiste en aplicar un algoritmo de inundación (flood fill) [1] similar al que se utiliza para colorear una región de una imagen. Para poder aplicar el algoritmo, se requiere que el usuario indique manualmente las coordenadas de un punto que pertenezca a la región libre del mapa (o, si hubiese varias regiones libres desconectadas entre sí, un punto para cada una de ellas). La figura 4b muestra el resultado tras este último paso.

\subsection{Reducción de los Conjuntos de Datos}

Según avanza la simulación de la dispersión del gas, se va registrando el estado del entorno (viento y concentración del gas en cada celda) en cada instante de tiempo. Esto es lo que permite recrear la simulación posteriormente de manera rápida, pero requiere espacio para almacenar estos resultados, lo que puede ser problemático en caso de tratar entornos muy grandes o de tener que trabajar con múltiples simulaciones.

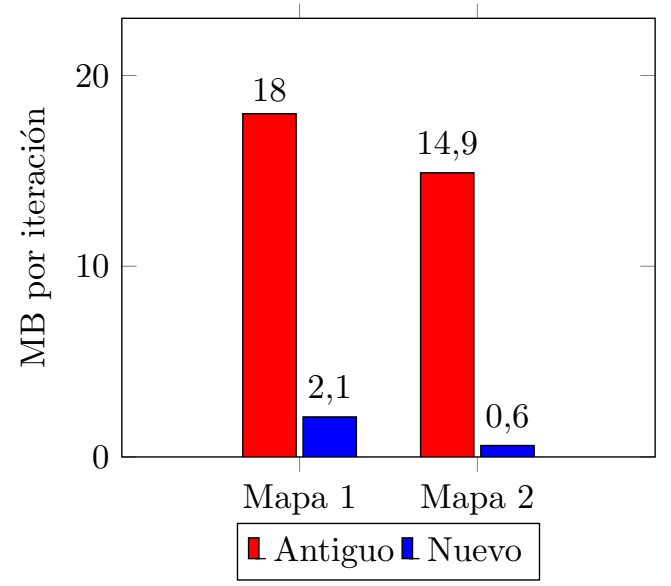

Figura 5: Comparativa de la memoria utilizada.
Para reducir el tamaño de los conjuntos de datos generados por las simulaciones se ha integrado en el proceso de simulación una etapa de compresión de los conjuntos de datos. También se ha modificado el formato de los archivos generados para reducir su tamaño, evitando escribir datos innecesarios, como los datos de viento y concentración de gas en las celdas ocupadas, en las que siempre van a ser inexistentes; y especificando el orden de magnitud de los valores una sola vez al principio del archivo, lo que permite expresar cada uno de los valores de manera más concisa.

La figura 5 muestra la reducción del tamaño de los conjuntos de datos generados para los mapas utilizados en las pruebas.

\subsection{Paralelización de la Simulación de Filamentos}

Dado que la simulación es inherentemente compleja, y con el añadido del proceso de compresión de los datos, se han hecho necesarios algunos ajustes al proceso para aumentar su rendimiento.

La simulación de la dispersión del gas se basa en el modelo de filamentos [4]. Cada uno de los filamentos de gas se mueve y expande de acuerdo a los valores de viento a los que es expuesto, y está modelado como una distribución normal de moléculas en 3D.

Aprovechando la naturaleza del modelo de dispersión utilizado, en el que los filamentos se mueven de manera independiente unos de otros, se ha paralelizado el cálculo del movimiento de cada filamento, parte central de la simulación.

También se realiza de manera paralelizada el cálculo del aporte de cada uno de los filamentos a la concentración de gas de las celdas. En la figura 6 se puede observar como el impacto de este cambio se hace notable a medida que la simulación avanza y el número de filamentos presentes crece.

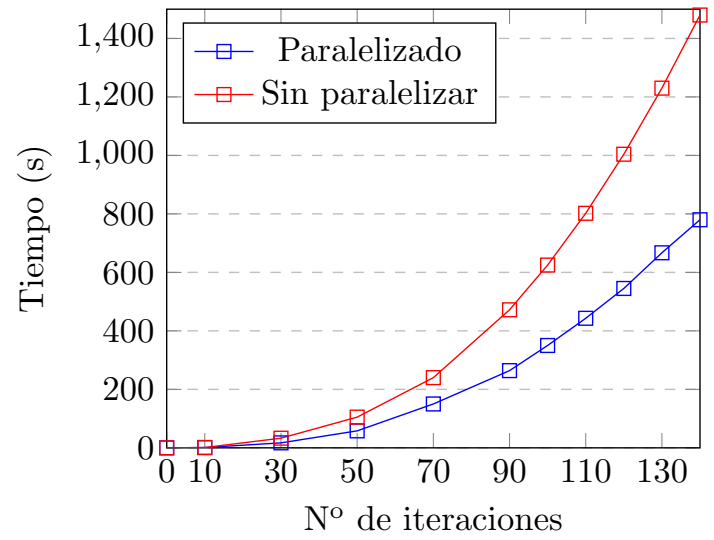

Figura 6: Datos experimentales del rendimiento. 


\subsection{Conclusiones}

En este trabajo se ha presentado una serie de modificaciones al simulador GADEN que mejoran su eficiencia tanto en el tiempo requerido para la ejecución de las simulaciones como en la memoria utilizada por los datos generados. También se ha incluido un algoritmo para generar un mapa de ocupación tridimensional a partir de modelos CAD, que ha sido diseñado para GADEN pero puede ser utilizado para otras aplicaciones similares.

Pese a todo esto, aún quedan aspectos a mejorar en el simulador, siendo uno de los más interesantes modelar el efecto del movimiento de un robot sobre el flujo de los gases en la simulación. GADEN es un proyecto de código abierto, y todos los materiales (código, tutoriales, etc) están disponibles en el sitio web del proyecto ${ }^{5}$.

\section{Agradecimientos}

Este trabajo se ha desarrollado en el marco del proyecto WISER (DPI2017-84827-R), financiado por el Ministerio de Ciencia e Innovación contando con fondos del Fondo Europeo de Desarrollo Regional (FEDER).

\section{English summary \\ IMPROVEMENTS TO THE GAS DISPERSION SIMULATOR GA- DEN FOR ROBOTIC OLFACTION}

Abstract. Within scientific disciplines, simulation tools play a fundamental role during the process of designing and developing new solutions, reducing development times and enabling detailed studies of the effect of multiple parameters. In this work we focus on GADEN, a novel gas dispersion simulator for robotic applications, proposing a set of improvements with the aim to facilitate its use and improve its computational performance. In addition to a detailed description of the changes introduced, a quantitative study of their effect on the performance of the simulation is included.

Keywords: GADEN, gas dispersion, simulation, mobile robotics olfaction, plume tracking, gas source localization, gas distribution mapping.

\section{Referencias}

[1] Sergei Burtsev and Ye.P. Kuzmin. An efficient flood-filling algorithm. Computers \& Graphics, 17:549-561, 091993.

[2] Gonçalo Cabrita, Pedro Sousa, and Lino Marques. Player/stage simulation of olfactory experiments. pages 1120 - 1125, 112010.

\footnotetext{
${ }^{5}$ http://mapir.isa.uma.es/work/gaden
}

[3] Saverio De Vito, Ettore Massera, M Piga, L Martinotto, and G Di Francia. On field calibration of an electronic nose for benzene estimation in an urban pollution monitoring scenario. Sensors and Actuators B: Chemical, 129(2):750-757, 2008.

[4] Jay A. Farrell, John Murlis, X. Long, Wei Li, and Ring T. Cardé. Filament-based atmospheric dispersion model to achieve short time-scale structure of odor plumes. 2002.

[5] Javier Gonzalez-Jimenez, Javier Monroy, and José-Luis Blanco. The multi-chamber electronic nose-an improved olfaction sensor for mobile robotics. Sensors, 11(6):6145-6164, 2011.

[6] Sava Grozdev and Deko Dekov. Barycentric coordinates: Formula sheet. International Journal of Computer Discovered Mathematics, 1:72-82, 03 2016.

[7] Michael Hutchinson, Cunjia Liu, and Wen Hua Chen. Information-Based Search for an Atmospheric Release Using a Mobile Robot: Algorithm and Experiments. IEEE Transactions on Control Systems Technology, pages 1-15, 2018.

[8] H. Ishida, Y. Wada, and H. Matsukura. Chemical sensing in robotic applications: A review. IEEE Sensors Journal, 12(11):3163-3173, Nov 2012.

[9] Paul Keller, Lars J. Kangas, Lars Liden, Sherif Hashem, and Richard Kouzes. Electronic noses and their applications. Proc. IEEE Technical Applications Conference and Workshops Northcon, 121995.

[10] Ji Gong Li, Qing Hao Meng, Yang Wang, and Ming Zeng. Odor source localization using a mobile robot in outdoor airflow environments with a particle filter algorithm. Autonomous Robots, 30(3):281-292, 2011.

[11] Amy Loufti, Silvia Coradeschi, Achim Lilienthal, and Javier Gonzalez-Jimenez. Gas distribution mapping of multiple odour sources using a mobile robot. Robotica, 27:311-319, 2009.

[12] C.W. Miller and L.M. Hively. A review of validation studies for the gaussian plume atmospheric dispersion model. Nucl. Saf.; (United States).

[13] Javier Monroy, José-Luis Blanco, and Javier Gonzalez-Jimenez. An open source framework for simulating mobile robotics olfaction (abstract only). In 15th International Symposium on Olfaction and Electronic Nose (ISOEN), 2013.

[14] Javier Monroy, José-Luis Blanco, and Javier Gonzalez-Jimenez. Time-variant gas distribution mapping with obstacle information. Autonomous Robots, 40(1):1-16, 2016.

[15] Javier Monroy and Javier González-Jiménez. Gas classification in motion: An experimental analysis. Sensors \& Actuators: B. Chemical, 2016.

[16] Javier Monroy and Javier Gonzalez-Jimenez. Towards Odor-Sensitive Mobile Robots, pages 244263. IGI Global, 2018.

[17] Javier Monroy, Javier Gonzalez-Jimenez, and José-Luis Blanco. Overcoming the slow recovery 
of mox gas sensors through a system modeling approach. Sensors, 12(10):13664-13680, oct 2012.

[18] Javier Monroy, Victor Hernandez-Bennetts, Han Fan, Achim Lilienthal, and Javier GonzalezJimenez. GADEN: A 3D gas dispersion simulator for mobile robot olfaction in realistic environments. Sensors (Switzerland), 17(7):1-16, 2017.

[19] Javier Monroy, Mariano Jaimez, and Javier Gonzalez-Jimenez. Online estimation of $2 \mathrm{~d}$ wind maps for olfactory robots. In International Symposium on Olfaction and Electronic Nose (ISOEN), pages 1-3, 2017.

[20] Javier Monroy, J. R. Ruiz-Sarmiento, FranciscoAngel Moreno, Francisco Melendez-Fernandez, Cipriano Galindo, and Javier Gonzalez-Jimenez. A semantic-based gas source localization with a mobile robot combining vision and chemical sensing. Sensors, 18(12), 2018.

[21] Brian P. Gerkey, Richard Vaughan, and Andrew Howard. The player/stage project: Tools for multi-robot and distributed sensor systems. Proceedings of the International Conference on $A d$ vanced Robotics, 082003.

[22] M. Reggente and A. J. Lilienthal. Using local wind information for gas distribution mapping in outdoor environments with a mobile robot. In SENSORS, 2009 IEEE, pages 1715-1720, Oct 2009.

[23] Frank-Michael Schleif, Barbara Hammer, Javier Gonzalez Monroy, Javier Gonzalez Jimenez, JoseLuis Blanco-Claraco, Michael Biehl, and Nicolai Petkov. Odor recognition in robotics applications by discriminative time-series modeling. Pattern Analysis and Applications, 18(1), 12015.

[24] Spyros Sklavounos and Fotis Rigas. Validation of turbulence models in heavy gas dispersion over obstacles. Journal of Hazardous Materials, 108(12):9-20, apr 2004.

[25] Carlos Sánchez-Garrido, Javier Monroy, and Javier Gonzalez-Jimenez. A configurable smart enose for spatio-temporal olfactory analysis. In IEEE Sensors, pages 1968-1971, oct 2014.

[26] Carlos Sánchez-Garrido, Javier Monroy, and Javier Gonzalez-Jimenez. Probabilistic Estimation of the Gas Source Location in Indoor Environments by Combining Gas and Wind Observations, pages 110-121. IOS Press, 2018.

[27] S. M. Tauseef, D. Rashtchian, and S. A. Abbasi. CFD-based simulation of dense gas dispersion in presence of obstacles. Journal of Loss Prevention in the Process Industries, 24(4):371-376, jul 2011.

[28] Yuta Wada, Marco Trincavelli, Yuichiro Fukazawa, and Hiroshi Ishida. Collecting a database for studying gas distribution mapping and gas source localization with mobile robots. 102010.

[29] Lei Zhang, Fengchun Tian, Hong Nie, Lijun Dang, Guorui Li, Qi Ye, and Chaibou Kadri. Classification of multiple indoor air contaminants by an electronic nose and a hybrid support vector machine. Sensors and Actuators B: Chemical, 174:114-125, 2012.

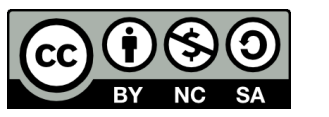

(C) 2019 by the authors. Submitted for possible open access publication under the terms and conditions of the Creative Commons Attribution CC BY-NC-SA 4.0 license (https://creativecommons.org/licenses/by-ncsa/4.0/deed.es). 